\title{
Robust Fault Detection and Isolation of Wind Turbines using Interval Observers
}

\author{
Joaquim Blesa, Fatiha Nejjari, Damiano Rotondo and Vicenç Puig
}

\begin{abstract}
In order to improve reliability and safety of wind turbines, it is important to detect and isolate faults as fast as possible. In this work, an approach based on interval observers is used for robust Fault Detection and Isolation (FDI) of wind turbines. Fault detection is addressed using interval observers and unknown but bounded descriptions of the noise and modeling errors, while fault isolation is based on a rowreasoning approach. The performance of the proposed robust FDI scheme is assessed using a set of fault scenarios considered in a wind turbine benchmark.
\end{abstract}

\section{INTRODUCTION}

Wind energy is inexhaustible, widely distributed and clean. These factors have made it fast growing in recent years. Wind turbines need to operate reliably at all times. Unfortunately, some faults that can occur in the gearbox, blades, sensors or actuators, could cause remarkable downtime of the wind turbine. Therefore, the design of fault diagnosis techniques is a crucial step in achieving reliable operations of them. If faults can be detected and identified at an early point, the consequences in breakdown, damages and repair costs will be less.

FDI algorithms can be used to detect the faults in the the wind turbine and accommodate them when possible. Model-based FDI is necessary to obtain a good diagnosis of faults. The generated residuals must be sensitive to the faults of interest and insensitive to process and measurement noises. The problem of model-based fault diagnosis in wind turbines has been recently addressed. Methods ranging from Kalman filters [1], observers [2] or parity equations [3] have been suggested as possible model-based techniques for fault diagnosis of wind turbines.

In [2], a benchmark model for fault detection and isolation as well as fault tolerant control of wind turbines is proposed. The benchmark model describes a realistic generic three blade horizontal variable speed wind turbine with a full scale converter coupling and a rated power of $4.8 \mathrm{MW}$. The high noise on the wind speed measurement, nonlinearities in the aerodynamic torque and uncertainties make the FDI a challenging problem. Some solutions to FDI in this benchmark model were proposed in an FDI benchmark competition. In particular, in [4], both an observer-based

This work has been funded by the Spanish MINECO through the project CYCYT SHERECS (ref. DPI2011-26243), by the European Commission through contract i-Sense (ref. FP7-ICT-2009-6-270428) and by UPC through the grant FPI-UPC E-01104 and by AGAUR through the contract FI-DGR 2013 (ref. 2013FIB00218).

Joaquim Blesa, Fatiha Nejjari, Damiano Rotondo and Vicenç Puig are with Advanced Control Systems Group (SAC), Universitat Politècnica de Catalunya (UPC), Rambla de Sant Nebridi, 11, 08222 Terrassa, Spain. e-mail: joaquim.blesa@upc.edu and a Kalman filter-based approach have been applied for both residual generation and evaluation, the latter performed taking into account the statistical properties of the residual signals. For fault isolation, a bank of residual generators based on dual sensor redundancy has been used. In [5], a set-membership approach for the FDI of the Wind Turbine Benchmark was proposed. The scheme was based on the use of parity equations for residual generation and the on-line analysis of the observed fault signatures for fault isolation. On the other hand, an FDI strategy based on piecewise affine Takagi Sugeno models was presented in [6]. A method that used both physical and analytical redundancy for residual generation and an up-down counter/up-down counters for fault decision was proposed by [7]. Recently, in [8], a setmembership approach for fault detection of the benchmark wind turbine has been proposed.

In this paper, the problem of robust fault diagnosis in wind turbines is addressed applying the interval observerbased approach proposed in [9]. The proposed model-based fault detection methodology relies on the use of observers and unknown but bounded descriptions of the noise and modeling errors. Robustness is achieved by propagating the model uncertainty to the residual alarm limit generating an adaptive threshold. When the residuals are outside the alarm limits it is argued that model uncertainty alone cannot explain the residual; and therefore, a fault must have occurred. Fault isolation is based on analyzing the observed fault signatures on-line and matching them with the theoretical ones, obtained by means of the structural analysis, using a row-reasoning scheme. The performance of the proposed FDI scheme is tested on the benchmark model [2] considering some fault scenarios.

In Section II, the proposed robust fault detection and isolation based on interval observers as well as a method for estimating parameter uncertainty are presented. In Section III, the wind turbine is briefly introduced and the set of residuals that will be used for fault diagnosis is generated. The results obtained by applying the proposed robust FDI approach to the wind turbine benchmark are presented in Section IV. Finally, some conclusions are given in Section V.

\section{Fault Detection, Isolation and Uncertainty Estimation}

\section{A. Problem set-up}

Let us consider that the wind turbine to be monitored can be described by a MIMO linear uncertain dynamic model 
expressed as follows:

$$
\begin{aligned}
x(k+1) & =A(\tilde{\theta}) x(k)+B(\tilde{\theta}) u(k)+F_{a}(\tilde{\theta}) f_{a}(k) \\
y(k) & =C(\tilde{\theta}) x(k)+F_{y}(\tilde{\theta}) f_{y}(k)+\tilde{v}(k)
\end{aligned}
$$

where $u(k) \in \mathbb{R}^{n_{u}}$ is the system input, $y(k) \in \mathbb{R}^{n_{y}}$ is the system output, $x(k) \in \mathbb{R}^{n_{x}}$ is the state-space vector, $\tilde{v}(k) \in \mathbb{R}^{n_{y}}$ is the output noise that is assumed to be bounded $|\tilde{v}(k)|<$ $\sigma, f_{a}(k) \in \mathbb{R}^{n_{u}}$ and $f_{y}(k) \in \mathbb{R}^{n_{y}}$ represent faults in the actuators and sensors, respectively. $A(\tilde{\theta}), B(\tilde{\theta}), C(\tilde{\theta}), F_{a}(\tilde{\theta})$ and $F_{y}(\tilde{\theta})$ are matrices of appropriate dimensions, where $\tilde{\theta}$ is the parameters vector.

The system (1)-(2) is monitored using a linear observer with Luenberger structure that uses an interval model of the system, i.e. a model with parameters bounded by intervals:

$$
\theta \in \Theta=\left\{\theta \in \mathbb{R}^{n_{\theta}} \mid \underline{\theta} \leq \theta \leq \bar{\theta}\right\}
$$

that represent the uncertainty about the exact knowledge of real parameters $\tilde{\theta}^{1}$. This observer, known as interval observer, is expressed as follows [12]:

$$
\begin{aligned}
\hat{x}(k+1, \theta) & =A_{0}(\theta) \hat{x}(k, \theta)+B(\theta) u(k)+L y(k) \\
\hat{y}(k, \theta) & =C(\theta) \hat{x}(k, \theta)
\end{aligned}
$$

where $\hat{x}(k, \theta)$ is the estimated system state vector, $\hat{y}(k, \theta)$ is the estimated system output vector and $A_{0}(\theta)=A(\theta)-L C(\theta)$ is the observer matrix.

The observer gain matrix $L \in \mathbb{R}^{n_{x} \times n_{y}}$ is designed to stabilize the matrix $A_{0}(\theta)$ and to guarantee a desired performance regarding fault detection, for all $\theta \in \Theta$, using the LMI pole placement approach [13].

The input/output form of the system (1)-(2) using the shift operator $q^{-1}$ and assuming zero initial conditions is given by:

$$
\begin{aligned}
y(k)=G_{u}\left(q^{-1}, \tilde{\theta}\right) u(k) & +G_{f_{a}}\left(q^{-1}, \tilde{\theta}\right) f_{a}(k)+F_{y}(\tilde{\theta}) f_{y}(k)+\tilde{v}(k) \\
G_{u}\left(q^{-1}, \tilde{\theta}\right) & =C(\tilde{\theta})(q I-A(\tilde{\theta}))^{-1} B(\tilde{\theta}) \\
G_{f_{a}}\left(q^{-1}, \tilde{\theta}\right) & =C(\tilde{\theta})(q I-A(\tilde{\theta}))^{-1} F_{a}(\tilde{\theta})
\end{aligned}
$$

Thus, the input/output form of the observer (4) is expressed as follows:

$$
\hat{y}(k, \theta)=G\left(q^{-1}, \theta\right) u(k)+H\left(q^{-1}, \theta\right) y(k)
$$

with:

$$
\begin{aligned}
& G\left(q^{-1}, \theta\right)=C(\theta)\left(q I-A_{0}(\theta)\right)^{-1} B(\theta) \\
& H\left(q^{-1}, \theta\right)=C(\theta)\left(q I-A_{0}(\theta)\right)^{-1} L
\end{aligned}
$$

The effect of the uncertain parameters $\theta$ on the observer temporal response $\hat{y}(k, \theta)$ will be bounded using an interval satisfying:

$$
\hat{y}(k, \theta) \in[\underline{\hat{y}}(k), \overline{\hat{y}}(k)]
$$

Such interval can be computed independently for each output $i\left(i=1, \ldots, n_{y}\right)$, neglecting couplings among outputs, as follows:

$$
\underline{\hat{y}_{i}}(k)=\min _{\theta \in \Theta} \hat{y}_{i}(k, \theta) \quad \text { and } \quad \overline{\hat{y}}_{i}(k)=\max _{\theta \in \Theta} \hat{y}_{i}(k, \theta)
$$

\footnotetext{
${ }^{1}$ The intervals for uncertain parameters can be inferred from real data using set-membership parameter estimation algorithms [10], [11].
}

subject to the observer equations given by (4). The interval bounds in (12) are determined using a method based on zonotopes [14], that is more efficient than other numerical optimisation methods, from a computational point of view. Finally, taking into account that the additive noise in the system (2) is bounded, the following condition should be satisfied

$$
y(k) \in[\underline{\hat{y}}(k)-\sigma, \overline{\hat{y}}(k)+\sigma]
$$

in a non-faulty scenario.

\section{B. Parameter uncertainty estimation}

Classical system identification methods [15] are formulated under a statistical framework. Assuming that the measured variables are corrupted by additive noises with known statistical distributions and that the model structure is known, a parameter estimation algorithm will provide nominal values for the parameters together with descriptions of the associated uncertainty in terms of the covariance matrix or confidence regions for a given probability level [16], [17]. However, this type of approaches cannot be applied when measurement errors are described as unknown but bounded values and/or when modeling errors exist.

Recently, some methodologies that provide a model with its uncertainty have been developed [18]. One of these methodologies, known as bounded-error set-membership estimation [10], assumes a bounded but unknown description of the noise and parametric uncertainty and produces a set of parameters consistent with the model structure. This approach will be used in this work to estimate the parametric uncertainty of the interval observer in (4).

Regarding the uncertain variables that appear in (4), it is assumed that a priori considerations, theoretical or practical, allow to obtain useful intervals associated to measurement noises, leading to an estimation of the noise bound $\sigma$. The goal of the parameter estimation algorithm is to characterize the parameter set $\Theta$, chosen in this paper as a box, consistent with the data collected in a fault-free scenario. Given $N$ measurements of system inputs $u(k)$ and outputs $y(k)$ from a scenario free of faults and rich enough from the identifiability point of view and a nominal model described by a vector $\theta_{n}$ obtained using a least-square parameter estimation algorithm [15], the uncertain parameter estimation algorithm proceeds by solving the following optimization problem

$\min \alpha$

subject to :

$$
\begin{aligned}
& y(k) \in[\hat{y}(k)-\sigma, \overline{\hat{y}}(k)+\sigma] \quad k=1, \ldots, N \\
& \hat{y}(k)=\min _{\theta \in \Theta} \hat{y}(k, \theta) \quad k=1, \ldots, N \\
& \overline{\overline{\hat{y}}}(k)=\max _{\theta \in \Theta} \hat{y}(k, \theta) \quad k=1, \ldots, N \\
& \hat{y}(k, \theta)=G\left(q^{-1}, \theta\right) u(k)+H\left(q^{-1}, \theta\right) y(k) \quad k=1, \ldots, N \\
& \Theta=\left[\theta_{n}(1-\alpha), \theta_{n}(1+\alpha)\right]
\end{aligned}
$$

\section{Fault detection test}

Fault detection test is based on generating nominal residuals comparing the measurements of some physical system 
variables $y(k)$ with their estimation $\hat{y}(k)$ provided by the observer (4):

$$
r(k)=y(k)-\hat{y}\left(k, \theta_{n}\right)
$$

where $r(k) \in \mathbb{R}^{n_{y}}$ is the residual set and $\theta_{n}$ the nominal parameters. According to [19], the computational form of the nominal residual generator, obtained using (4), is:

$$
r(k)=\left(I-H\left(q^{-1}, \theta_{n}\right)\right) y(k)-G\left(q^{-1}, \theta_{n}\right) u(k)
$$

that has been derived taking into account the input/output form of the observer (8).

When considering model uncertainty located in parameters, the residual generated by (15) will not be zero, even in a non-faulty scenario. To cope with the effect of parameter uncertainty effect, a passive robust approach based on adaptive thresholding can be used [9]. Thus, using this passive approach, the effect of parameter uncertainty in the residual $r(k)$ is bounded by the interval [20]:

$$
r(k) \in[\underline{r}(k)-\sigma, \bar{r}(k)+\sigma]
$$

where:

$$
\underline{r}(k)=\underline{\hat{y}}(k)-\hat{y}\left(k, \theta_{n}\right) \text { and } \bar{r}(k)=\overline{\hat{y}}(k)-\hat{y}\left(k, \theta_{n}\right)
$$

where $\hat{y}(k)$ and $\overline{\hat{y}}(k)$ are the bounds of the system output estimation computed component-wise using the interval observer (4) and obtained according to (12).

Then, the fault detection test could be based on checking if the residuals satisfy or not the condition given by (17). In case that this condition does not hold, a fault can be indicated.

\section{Fault isolation test}

Fault isolation consists in identifying the faults affecting the system. It is carried out on the basis of fault signatures, generated by the detection module, and their relation with all the considered faults, $f(k)=\left\{f_{a}(k), f_{y}(k)\right\}$. Robust residual evaluation presented in Section II-C allows obtaining a set of fault signatures $\phi(k)=\left[\phi_{1}(k), \phi_{2}(k), \ldots, \phi_{n y}(k)\right]$, where each fault indicator is given by:

$$
\phi_{i}(k)=\left\{\begin{array}{lll}
0 & \text { if } & r_{i}(k) \notin\left[r_{i}(k)-\sigma, \overline{r_{i}}(k)+\sigma\right] \\
1 & \text { if } \quad r_{i}(k) \in\left[\underline{r_{i}}(k)-\sigma, \overline{r_{i}}(k)+\sigma\right]
\end{array}\right.
$$

Standard fault isolation reasoning exploits the knowledge about the binary relation that exists between the set of fault hypothesis and the set of residuals, stored in the so called Fault Signature Matrix (FSM), denoted as M. An element $m_{i j}$ of $M$ is equal to 1 if the fault $f^{j}$ affects the computation of the residual $r_{i}$; otherwise, the element $m_{i j}$ is zero-valued. A column of $M$ is known as a theoretical fault signature and indicates which residuals are affected by a given fault. If two or more columns are equal, the corresponding faults cannot be distinguished. Hence, a set of faults is isolable if all the columns in $M$ are different.

Due to the uncertainty, when a fault is present in the system, an undefined number of the residuals affected by the fault can be found inconsistent, mainly depending on the sensitivity of each residual with respect to the fault and on the fault magnitude. In other words, the observed fault

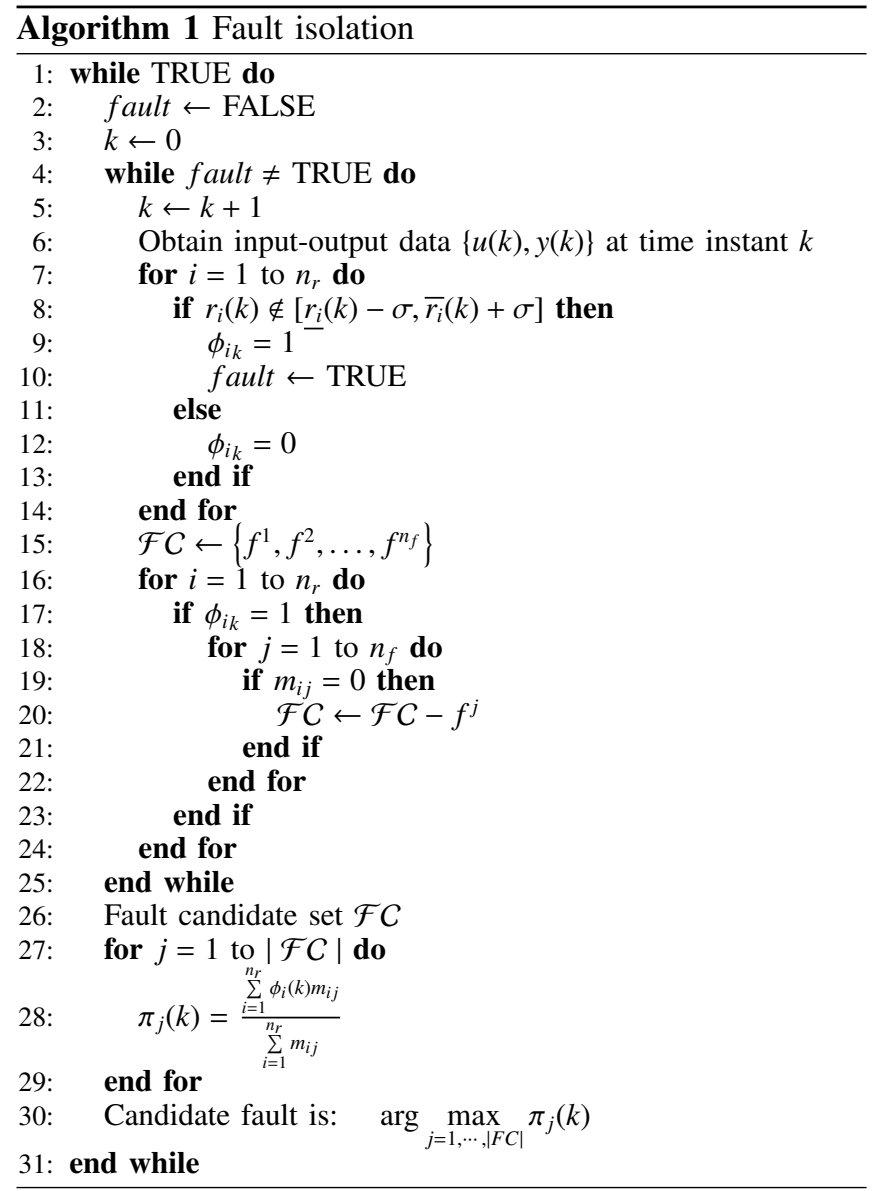

signature will not exactly match the theoretical signature of the present fault. In this case, if the column-matching procedure is used, then that particular fault will not be identified. An appropriate reasoning should only consider the residuals that are inconsistent when searching for the fault (inconsistency is relevant, consistency is not). A residual that is found inconsistent indicates that one of the faults that affects the residual is acting on the system. But the contrary is not true: if a residual is satisfied, it does not assure that none of the associated faults is present. According to the established terminology [21], the used algorithm must avoid single-fault exoneration, which is implicit in the column matching reasoning.

Under single-fault assumption, this can be easily achieved by taking into account that the fault that is actually present in the system has to affect all the residuals that have been found inconsistent according to the observed fault signature (if not, the single fault hypothesis cannot explain the observed behavior). Algorithm 1 summarizes an isolation procedure based on the row-reasoning approach.

\section{Case study: Wind Turbine System}

\section{A. Wind Turbine Model and Fault Scenarios}

A complete description of the wind turbine model of the FDI/FTC benchmark can be found in [2]. Such a model comprises the Wind model, the Blade and Pitch model, the Drive train model, the Generator/Converter model, the 
TABLE I: WT Faults Description

\begin{tabular}{cccc}
\hline Fault & Fault description & Type & Period \\
\hline$f_{1}$ & Pitch 1 measurement & Fixed value & 2000s-2100s \\
$f_{2}$ & Pitch 2 measurement & Gain factor & $2300 \mathrm{~s}-2400 \mathrm{~s}$ \\
$f_{3}$ & Pitch 3 measurement & Fixed value & $2600 \mathrm{~s}-2700 \mathrm{~s}$ \\
$f_{4}$ & Rotor speed sensor $\omega_{r}$ & Fixed value & $1500 \mathrm{~s}-1600 \mathrm{~s}$ \\
$f_{5}$ & Rotor and generator speed & Gain factor & \\
& measurements $\omega_{r}$ and $\omega_{g}$ & & $1000 \mathrm{~s}-1100 \mathrm{~s}$ \\
$f_{6}$ & Parameter abrupt change in pitch 2 & Changed dynamics & $2900 \mathrm{~s}-3000 \mathrm{~s}$ \\
$f_{7}$ & Parameter slowly change in pitch 3 & Changed dynamics & $3400 \mathrm{~s}-3500 \mathrm{~s}$ \\
$f_{8}$ & Offset in converter system & Offset & $3800 \mathrm{~s}-3900 \mathrm{~s}$ \\
\hline
\end{tabular}

Controller and the parameters of the models. Hereafter, only the Pitch system model and the Generator/Converter model will be shown, as the faults acting in the system affect those subsystems.

The hydraulic pitch system can be modeled as a second order transfer function:

$$
\frac{\beta(s)}{\beta_{r}(s)}=\frac{\omega_{n}^{2}}{s^{2}+2 \zeta \omega_{n} s+\omega_{n}^{2}}
$$

where $\beta(s)$ and $\beta_{r}(s)$ are the pitch angle and its reference, respectively, and $\omega_{n}$ and $\zeta$ are the natural frequency and the damping ratio of the pitch actuator model.

The converter dynamics can be modeled by a first order transfer function:

$$
\frac{\tau_{g}(s)}{\tau_{g, r}(s)}=\frac{\alpha_{g c}}{s+\alpha_{g c}}
$$

where $\tau_{g}$ and $\tau_{g, r}$ are the converter torque and its reference, respectively, and $\alpha_{g c}$ is the cutoff frequency.

The power produced by the generator depends on the rotational speed of the rotor and the applied load:

$$
P_{g}(t)=\eta_{g} \omega_{g}(t) \tau_{g}(t)
$$

where $P_{g}$ is the power produced by the generator, $\eta_{g}$ its efficiency and $\omega_{g}$ the rotational speed of the rotor.

Since the turbine has three blades, all three pitch positions are measured. Such a measurement is done with two sensors for each blade in order to ensure physical redundancy. The variables defined as $\beta_{r 1}, \beta_{r 2}, \beta_{r 3}$ for the pitch reference to blade 1,2 and $3 ; \beta_{1, m 1}, \beta_{1, m 2}, \beta_{2, m 1}, \beta_{2, m 2}, \beta_{3, m 1}, \beta_{3, m 2}$ are the pitch positions measurements starting from the two measurements for the blade 1 followed by the two measurements for blade 2, and blade 3 in the end. For the same reason, the generator and rotor speeds are also measured with two sensors. The two rotor speed measurements are defined as $\omega_{r, m 1}, \omega_{r, m 2}$, while the two generator speed measurements are defined as $\omega_{g, m 1}, \omega_{g, m 2}$. The proposed FDI scheme is tested on the WT benchmark model considering the faults described in Table I.

\section{B. Residual Generation}

By applying structural analysis [22] to the WT model provided in [2], a set of twelve residuals expressed as reduced observers in input-output form are obtained.
TABLE II: Fault signature matrix

\begin{tabular}{ccccccccc}
\hline $\mathrm{r}$ & $f_{1}$ & $f_{2}$ & $f_{3}$ & $f_{4}$ & $f_{5}$ & $f_{6}$ & $f_{7}$ & $f_{8}$ \\
\hline$r_{1}$ & & & & $\mathrm{x}$ & $\mathrm{x}$ & & & \\
$r_{2}$ & $\mathrm{x}$ & $\mathrm{x}$ & $\mathrm{x}$ & & $\mathrm{x}$ & $\mathrm{x}$ & $\mathrm{x}$ & $\mathrm{x}$ \\
$r_{3}$ & & & & & $\mathrm{x}$ & & & \\
$r_{4}$ & $\mathrm{x}$ & $\mathrm{x}$ & $\mathrm{x}$ & & $\mathrm{x}$ & $\mathrm{x}$ & $\mathrm{x}$ & $\mathrm{x}$ \\
$r_{5}$ & $\mathrm{x}$ & & & & & & & \\
$r_{6}$ & $\mathrm{x}$ & & & & & & & \\
$r_{7}$ & & $\mathrm{x}$ & & & & & & \\
$r_{8}$ & & $\mathrm{x}$ & & & & $\mathrm{x}$ & & \\
$r_{9}$ & & & $\mathrm{x}$ & & & & & \\
$r_{10}$ & & & $\mathrm{x}$ & & & & $\mathrm{x}$ & \\
$r_{11}$ & & & & & & & & $\mathrm{x}$ \\
$r_{12}$ & & & & & $\mathrm{x}$ & & & \\
\hline
\end{tabular}

$$
\begin{aligned}
r_{1}(k)= & \omega_{r, m 1}(k)-\omega_{r, m 2}(k) \\
r_{2}(k)= & \left(1-\frac{l_{21} q^{-1}}{1-\left(a_{21}-l_{21}\right) q^{-1}}\right) \omega_{r, m 2}(k)-\frac{b_{21} q^{-1} \tau_{r}(k)+c_{21} q^{-1} \tau_{g, m}(k)}{1-\left(a_{21}-l_{21}\right) q^{-1}} \\
r_{3}(k)= & \omega_{g, m 1}(k)-\omega_{g, m 2}(k) \\
r_{4}(k)= & \left(1-\frac{l_{41} q^{-1}}{1-\left(a_{41}-l_{41}\right) q^{-1}}\right) \omega_{g, m 2}(k)-\frac{b_{41} q^{-1} \tau_{r}(k)+c_{41} q^{-1} \tau_{g, m}(k)}{1-\left(a_{41}-l_{41}\right) q^{-1}} \\
r_{5}(k)= & \beta_{1, m 1}(k)-\beta_{1, m 2}(k) \\
r_{6}(k)= & \left(1-\frac{l_{61} q^{-1}+l_{62} q^{-2}}{1-\left(a_{61}-l_{61}\right) q^{-1}-\left(a_{62}-l_{62}\right) q^{-2}}\right) \beta_{1, m 2}(k) \\
& -\frac{\left(b_{61} q^{-1}+b_{62} q^{-2}\right) \beta_{r}(k)}{1-\left(a_{61}-l_{61}\right) q^{-1}-\left(a_{62}-l_{62}\right) q^{-2}} \\
r_{7}(k)= & \beta_{2, m 1}(k)-\beta_{2, m 2}(k) \\
r_{8}(k)= & \left(1-\frac{l_{81} q^{-1}+l_{82} q^{-2}}{1-\left(a_{81}-l_{81}\right) q^{-1}-\left(a_{82}-l_{82}\right) q^{-2}}\right) \beta_{2, m 2}(k) \\
& -\frac{\left(b_{81} q^{-1}+b_{82} q^{-2}\right) \beta_{r}(k)}{1-\left(a_{81}-l_{81}\right) q^{-1}-\left(a_{82}-l_{82}\right) q^{-2}} \\
r_{9}(k)= & \beta_{3, m 1}(k)-\beta_{3, m 2}(k) \\
r_{10}(k) \quad= & \left(1-\frac{l_{101} q^{-1}+l_{102} q^{-2}}{1-\left(a_{101}-l_{101}\right) q^{-1}-\left(a_{102}-l_{102}\right) q^{-2}}\right) \beta_{3, m 2}(k) \\
& -\frac{\left(b_{101} q^{-1}+b_{102} q^{-2}\right) \beta_{r}(k)}{1-\left(a_{101}-l_{101}\right) q^{-1}-\left(a_{102}-l_{102}\right) q^{-2}} \\
r_{11}(k)= & \left(1-\frac{l_{111} q^{-1}}{1-\left(a_{111}-l_{111}\right) q^{-1}}\right) \tau_{g, m}(k)-\frac{b_{111} q^{-1} \tau_{g, r}(k)}{1-\left(a_{111}-l_{111}\right) q^{-1}} \\
r_{12}(k)= & P_{g, m}(k)-\eta_{g} \omega_{g, m 2} \tau_{g, m}
\end{aligned}
$$

where $a_{i j}, b_{i j}$ and $c_{i j}$ are model parameters that have to be estimated, $\eta_{g}$ is a known coefficient, and $l_{i j}$ are the observer gains.

Moreover, the FSM represented in Table II gives the relation between residuals and faults. A cross ' $x$ ' in the ith row and $j$-th column of the FSM indicates that the $i$-th residual is affected by the $\mathrm{j}$-th fault. Consequently $m_{i j}=1$ where there is a cross and $m_{i j}=0$ elsewhere.

\section{Uncertain parameter estimation}

Residuals $r_{1}, r_{3}, r_{5}, r_{7}$ and $r_{9}$ are static equations that involve the comparison of the values of two different sensors measuring the same variable. In the same way, residual $r_{12}$ compares the value of a sensor with the one calculated using a static relation with two other sensors. Residuals $r_{2}$ and $r_{4}$ are obtained from the discretization of the drive train model expressed in state space form. Residuals $r_{6}, r_{8}$ and $r_{10}$ follow the discretization of the continuous second transfer function that models the hydraulic pitch system. Finally, residual $r_{11}$ 
is derived from the discretization of the first order transfer function that models the converter dynamics.

In order to reduce the effect of the noise in fault detection and isolation procedures, the variables involved in the discretized regressor equations have been filtered by second order low-pass filters. Nominal coefficients of dynamical residuals have been obtained applying the least square method to the data obtained simulating the system in the fault free scenario.

The parameter estimation procedure described in Section II-B has been applied to the fault-free scenario specified in the benchmark in order to obtain the intervals for the parameters of the residuals that will be used for fault detection and isolation purposes. The noise bound $\sigma$ is estimated using three times the standard deviation of the nominal residual (15).

\section{Fault Detection and Isolation}

The fault detection has been implemented by means of (17) applied to the 12 residuals obtained in Section III-B. The fault isolation has been implemented using the Algorithm 1 with the FSM presented in Table II.

\section{Results}

The proposed robust FDI scheme has been evaluated with the benchmark model and the faults provided by [2]. Due to lack of space only the results of two fault scenarios are shown in this work. Fig. 1 and Fig. 2 show the nominal residuals $r_{1}^{0}, \ldots, r_{12}^{0}$ and the observed fault signature components $\phi_{1}, \ldots, \phi_{12}$ used in the proposed fault detection and isolation procedure in fault scenario 3 (fixed value in the third pitch measurement from $t=2600 \mathrm{~s}$ to $t=2700 \mathrm{~s}$ ). The fault is detected and isolated at instant $t=2600.06 \mathrm{~s}$, as shown in Fig. 3. It can be seen that the FDI system satisfies the detection time requirements [2].
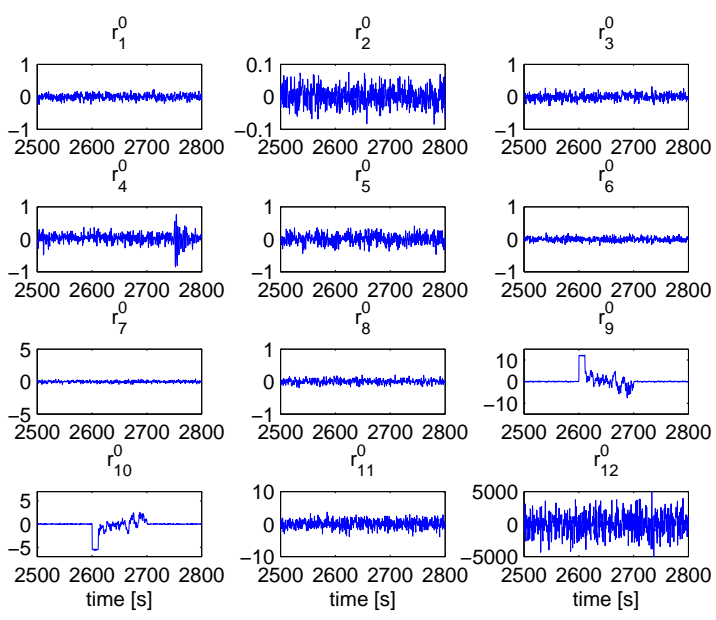

Fig. 1: Nominal residuals $r_{1}^{0}, \ldots, r_{12}^{0}$ in fault scenario 3 .

Fig. 4 and Fig. 5 show the nominal residuals $r_{1}^{0}, \ldots, r_{12}^{0}$ and the observed fault signature components $\phi_{1}, \ldots, \phi_{12}$ used in the proposed fault detection and isolation procedure in fault
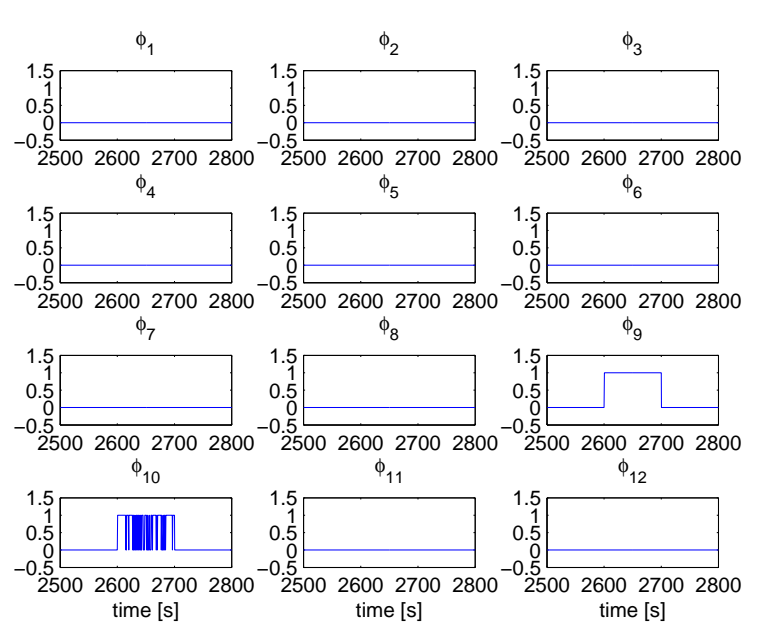

Fig. 2: Observed fault signature components in fault scenario 3.
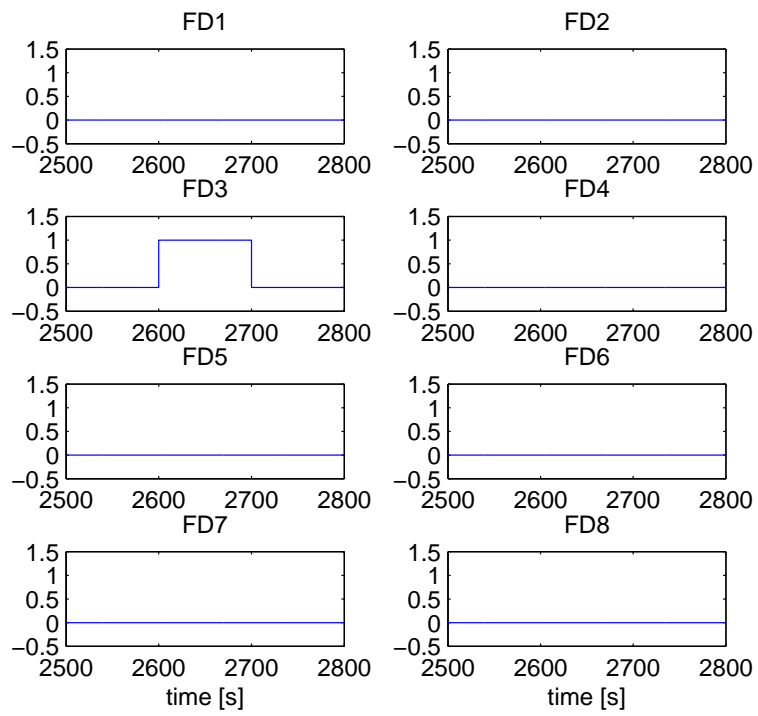

Fig. 3: Fault isolation results in fault scenario 3.

scenario 5 (change of gain in the rotor and generator speed measurement from $t=1000 \mathrm{~s}$ to $t=1100 \mathrm{~s})$. The fault $f_{5}$ is detected and isolated in 0.03 seconds $(t=1100.03 s)$, as shown in Fig. 6.

\section{Conclusions}

In this paper, a robust FDI scheme for wind turbines diagnosis has been proposed. The FDI scheme is based on interval observers, unknown but bounded description of the noise and modeling errors and a row-reasoning approach.The performance of the proposed FDI scheme has been assessed using the proposed fault scenarios considered in the wind turbine benchmark. Satisfactory results have been obtained for the different fault scenarios considered. 


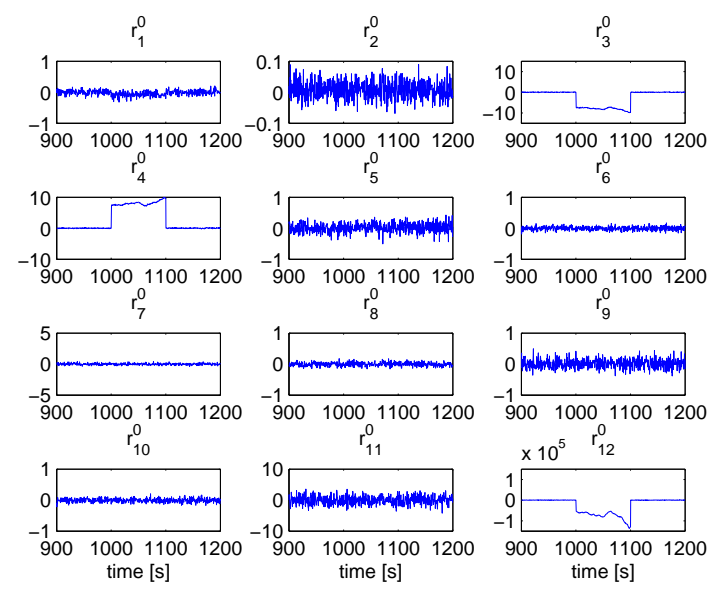

Fig. 4: Nominal residuals $r_{1}^{0}, \ldots, r_{12}^{0}$ in fault scenario 5 .

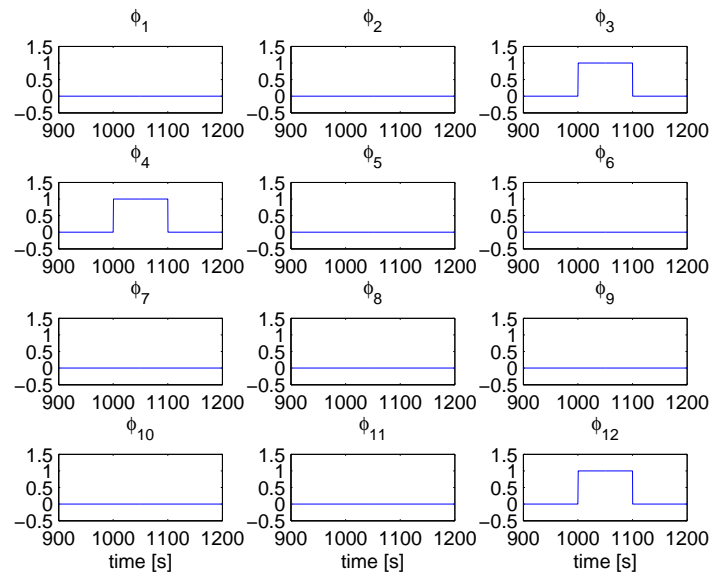

Fig. 5: Observed fault signature components in fault scenario 5.

\section{REFERENCES}

[1] X. Wei, M. Verhaegen, and T. van den Engelen, "Sensor fault diagnosis of wind turbines for fault tolerant," in Proceedings of 17th IFAC World Congress, Seoul, Korea, 2008.

[2] P. Odgaard, J. Stoustrup, and M. Kinnaert, "Fault tolerant control of wind turbines - a benchmark model," in Proceedings of the 7th Symposium on Fault Detection, Supervision and Safety of Technical Processes (SAFEPROCESS 2009), 2009, pp. 155-160.

[3] C. Dobrila and R. Stefansen, "Fault tolerant wind turbine control," Master's thesis, Technical University of Denmark, Kgl. Lyngby, Denmark., 2007.

[4] W. Chen, S. X. Ding, A. Haghani, A. Naik, A. Q. Khan, and S. Yin, "Observer-based FDI schemes for Wind Turbine Benchmark." in Proceedings of the 18th IFAC World Congress, Milan, Italy, 2011, pp. 7073-7078.

[5] J. Blesa, V. Puig, J. Romera, and J. Saludes, "Fault diagnosis of wind turbines using a set-membership approach." in Proceedings of the 18th IFAC World Congress, Milan, Italy, 2011, pp. 8316-8321.

[6] S. Simani, P. Castaldi, and A. Tilli, "Data-driven approach for wind turbine actuator and sensor fault detection and isolation," in Proceedings of the 18th IFAC World Congress, Milano, Italy, 2011, pp. 83018306.

[7] A. Ozdemir, P. Seiler, and G. Balas, "Wind turbine fault detection using counter-based residual thresholding," in Proceedings of IFAC World Congress 2011, 2011, pp. 8289-8294.
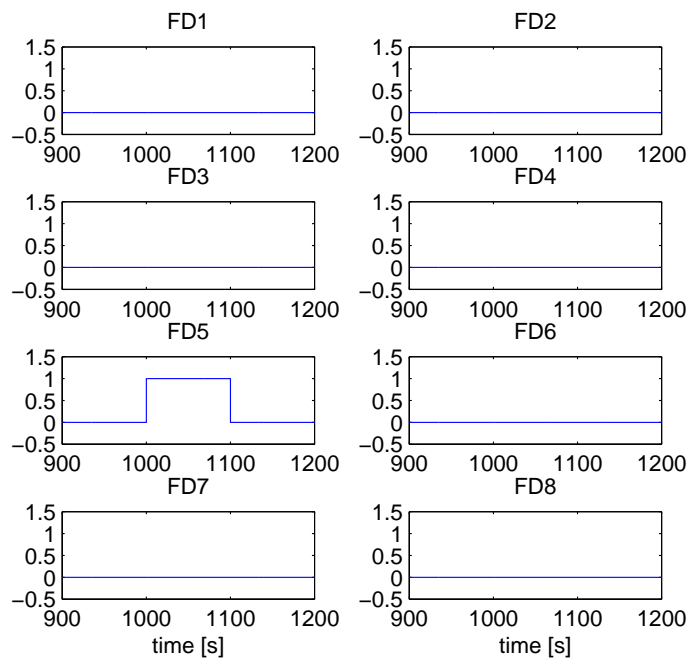

Fig. 6: Fault isolation results in fault scenario 5.

[8] S. M. Tabatabaeipour, P. F. Odgaard, T. Bak, and J. Stoustrup, "Fault Detection of Wind Turbines with Uncertain Parameters: A SetMembership Approach," Energies, vol. 5, no. 7, pp. 2424-2448, 2012.

[9] V. Puig, A. Stancu, T. Escobet, F. Nejjari, J. Quevedo, and R. J. Patton, "Passive Robust Fault Detection using Interval Observers: Application to the DAMADICS Benchmark Problem," Control Engineering Practice, vol. 14, no. 6, pp. 621-633, 2006.

[10] M. Milanese, J. Norton, H. Piet-Lahanier, and É. Walter, Eds., Bounding Approaches to System Identification. Springer, 1996.

[11] S. Ploix, O. Adrot, and J. Ragot, "Parameter Uncertainty Computation in Static Linear Models," Proceedings of the 38th IEEE Conference on Decision and Control, vol. 2, pp. 1916-1921, 1999.

[12] J. Meseguer, V. Puig, and T. Escobet, "Observer Gain Effect in Linear Interval Observer-based Fault Detection," Proceedings of the 6th IFAC Symposium on Fault Detection, Supervision and Safety of Technical Processes (SAFEPROCESSO6), 2006.

[13] M. Chilali and P. Gahinet, " $H_{\infty}$ Design with pole placement constraints: an LMI approach," IEEE Transactions on Automatic Control, vol. 41, no. 3, pp. 358-367, 1996.

[14] S. Montes de Oca, V. Puig, and J. Blesa, " Robust fault detection based on adaptive threshold generation using interval LPV observers," International Journal of Adaptive Control and Signal Processing, vol. 26, no. 3, pp. 258-283, 2012.

[15] L. Ljung, System Identification: Theory for the User. Englewood Cliffs, New Jersey: Prentice Hall, 1987.

[16] M. Kendall and A. Stuart, The Advanced Theory of Statistics. Charles Griffin, 1979, vol. 2.

[17] M. Dalai, E. Weyer, and M. Campi, "Parametric identification of nonlinear systems: guaranteed confidence regions," in Proceedings of the 44th IEEE Conference on Decision and Control, and the European Control Conference, 2005, pp. 6418-6423.

[18] W. Reinelt, A. Garulli, and L. Ljung, "Comparing different approaches to model error modeling in robust identification," Automatica, vol. 38, no. 5 , pp. 787-803, 2002

[19] J. Gertler, Fault Detection and Diagnosis in Engineering Systems. New York: Marcel Dekker, 1998

[20] V. Puig, J. Saludes, and J. Quevedo, "Worst-Case Simulation of Discrete Linear Time-Invariant Interval Dynamic Systems," Reliable Computing, vol. 9, no. 4, 2003.

[21] M. Cordier, P. Dague, F. Lévy, J. Montmain, M. Staroswiecki, and L. Travéé-Massuyès, "Conflicts versus analytical redundancy relations: A comparative analysis of the model based diagnosis approach from the artificial intelligence and automatic control perspectives," IEEE Transactions on Systems, Man, and Cybernetics - Part B: Cybernetics, vol. 34, no. 5, pp. 2163-2177, 2004.

[22] M. Blanke, M. Kinnaert, J. Lunze, and M. Staroswiecki, Diagnosis and Fault-Tolerant Control. $\quad$ Springer-Verlag Berlin Heidelberg, 2006. 